\title{
Pyrochlore-Type Compounds Containing Double Oxides of Trivalent and Tetravalent Ions ${ }^{1}$
}

\author{
Robert S. Roth
}

\begin{abstract}
A study has been made by X-ray diffraction analyses of the $\mathrm{A}_{2} \mathrm{O}_{3}: 2 \mathrm{BO}_{2}$-type double oxides. It was found that many of these mixed oxides, after appropriate heat treatment, formed compounds of the formula type $\mathrm{A}_{2} \mathrm{~B}_{2} \mathrm{O}_{7}$. Most of these compounds crystallized in the cubic system with a face-centered cell similar to that found for the mineral pyrochlore, although some were distorted from the ideal cubic structure.

Indexed X-ray diffraction powder patterns are given for the cubic compounds $\mathrm{Sm}_{2} \mathrm{O}_{3} \cdot 2 \mathrm{TiO}_{2}, \quad \mathrm{Gd}_{2} \mathrm{O}_{3} \cdot 2 \mathrm{TiO}_{2}, \quad \mathrm{Dy}_{2} \mathrm{O}_{3} \cdot 2 \mathrm{TiO}_{2}, \quad \mathrm{Y}_{2} \mathrm{O}_{3} \cdot 2 \mathrm{TiO}_{2}, \quad \mathrm{Yb}_{2} \mathrm{O}_{3} \cdot 2 \mathrm{TiO}_{2}, \quad \mathrm{La}_{2} \mathrm{O}_{3} \cdot 2 \mathrm{SnO}_{2}, \quad \mathrm{Nd}_{2} \mathrm{O}_{3}$. $2 \mathrm{SnO}_{2}, \mathrm{La}_{2} \mathrm{O}_{3} \cdot 2 \mathrm{ZrO} \mathrm{O}_{2}, \mathrm{Nd}_{2} \mathrm{O}_{3} \cdot 2 \mathrm{ZrO}$, and for the possible compounds $\mathrm{Y}_{2} \mathrm{O}_{3} \cdot 2 \mathrm{ZrO} \mathrm{O}_{2}$ and $\mathrm{Nd}_{2} \mathrm{O}_{3}$. $2 \mathrm{UO}_{2}$. Unindexed patterns are given for $\mathrm{La}_{2} \mathrm{O}_{3} \cdot 2 \mathrm{TiO}_{2}, \mathrm{Nd}_{2} \mathrm{O}_{3} \cdot 2 \mathrm{TiO}_{2}$ and $\mathrm{Bi}_{2} \mathrm{O}_{3} \cdot 2 \mathrm{SnO}_{2}$.

On the basis of the existence of the two compounds $\mathrm{La}_{2} \mathrm{O}_{3} \cdot 2 \mathrm{ZrO}_{2}$ and $\mathrm{Nd}_{2} \mathrm{O}_{3} \cdot 2 \mathrm{ZrO}_{2}$, the phase diagrams for the systems $\mathrm{La}_{2} \mathrm{O}_{3}-\mathrm{ZrO}_{2}$ and $\mathrm{Nd}_{2} \mathrm{O}_{3}-\mathrm{ZrO}_{2}$ have been revised.
\end{abstract}

\section{Introduction}

A partial survey of the reactions occurring in binary oxide mixtures of the type $\mathrm{A}_{2} \mathrm{O}_{3}: 2 \mathrm{BO}_{2}$ has been conducted as part of a program of fundamental research on ceramic materials. Combinations of simple oxides in this proportion were selected because of the current interest in ferroelectric ceramics. $\mathrm{Cd}_{2} \mathrm{Nb}_{2} \mathrm{O}_{7}$, at room temperature, has the cubic pyrochlore structure, although the ferroelectric form at low temperatures is distorted from this ideal structure $[1,2,3] .^{2}$ The fact that the compound $\mathrm{Cd}_{2} \mathrm{Nb}_{2} \mathrm{O}_{7}$ is known to be ferroelectric at certain temperatures [1] led to the study of other compounds with similar structure.

At the time this work was initiated, no references were known describing a pyrochlore structure that did not contain a pentavalent ion as an essential element of the compound. A ternary compound of this type having the formula $\mathrm{CaO} \cdot \mathrm{ZrO}_{2} \cdot 2 \mathrm{TiO}_{2}$ or $\left(\mathrm{Ca}_{1.0} \mathrm{Z}_{1.0}\right) \mathrm{Ti}_{2} \mathrm{O}_{7}$ was, however, described by Coughanour et al. [4]. Another reference has recently been made by Padrow and Schusterius [5] to pyrochlore phases in the system $\mathrm{La}_{2} \mathrm{O}_{3}-\mathrm{SnO}_{2}-\mathrm{TiO}_{2}$.

This report is concerned with some combinations of the oxides of trivalent and tetravalent ions which, on the basis of radius ratio, might be expected to form compounds of the pyrochlore type.

\section{Materials and Methods}

\subsection{Materials}

The following substances were used as sources of the component oxides in this study:

$\mathrm{TiO}_{2}$. A rutile of 99.5-percent purity.

$\mathrm{SnO}_{2}$. Precipitated tin oxide of over 98.5-percent purity.

$\mathrm{ZrO}_{2}$. Dense zirconia of over 99-percent purity, calcined at $1,450^{\circ} \mathrm{C}$.

1 This work has been sponsored as part of a program for Improvement of Piezoelectric Ceramics by the Office of Ordnance Research of the Department of the Army.

Figures in brackets indicate the literature references at the end of this paper.
$\mathrm{CeO}_{2}$. Calcined cerium dioxide of nominal 99-percent purity.

$\mathrm{UO}_{2}$. Urania, supplied by the United States Atomic Energy Commission, of over 99.9-percent purity.

$\mathrm{La}_{2} \mathrm{O}_{3}$. Lanthanum oxide of 98-percent purity, remainder mostly water and $\mathrm{CO}_{2}$.

$\mathrm{Nd}_{2} \mathrm{O}_{3}$. Neodymium oxide of over 99-percent purity.

$\mathrm{Sm}_{2} \mathrm{O}_{3}$. Samarium oxide of nominal 99-percent purity.

$\mathrm{Gd}_{2} \mathrm{O}_{3}$. Gadolinium oxide of nominal 98-percent purity.

$\mathrm{Bi}_{2} \mathrm{O}_{3}$. Bismuth trioxide of over 99-percent purity. $\mathrm{Bi}_{2} \mathrm{O}_{3} \cdot 3 \mathrm{SnO}_{2} \cdot 5 \mathrm{H}_{2} \mathrm{O}$. Precipitated hydrous bismuth stannate of at least 98-percent purity.

$\mathrm{Y}_{2} \mathrm{O}_{3}$. Yttrium oxide of 98- to 99-percent purity, the remainder being rare earths.

$\mathrm{Dy}_{2} \mathrm{O}_{3}$. Dysprosium oxide of nominal 98-percent purity.

$\mathrm{Yb}_{\mathbf{2}} \mathrm{O}_{3}$. Ytterbium oxide of nominal 98-percent purity.

$\mathrm{In}_{2} \mathrm{O}_{3}$. Rather impure indium oxide specified "for manufacturing use only."

$\mathrm{Sb}_{2} \mathrm{O}_{3}$. Antimony trioxide of over 99-percent purity, with 0.2 percent of $\mathrm{As}_{2} \mathrm{O}_{3}$.

\subsection{Sample Preparation}

The starting materials, in sufficient quantities to give either a $10.0 \mathrm{-g}$ sample or a $1.0 \mathrm{-g}$ sample, depending upon the availability of the raw materials, were weighed to the nearest milligram. No corrections were made for the percentage purity of the raw materials, except for loss due to water and carbon dioxide. They were then mixed together with a few drops of distilled water to assure bonding and formed into 1-in. or 1/2-in.-diam disks at a pressure of 5,000 $\mathrm{lb} / \mathrm{in}^{2}$. The pressed disks were fired for $4 \mathrm{hr}$ at $1,100^{\circ} \mathrm{C}$ on platinum foil in an air atmosphere, using an electrically heated furnace wound with $80 \% \mathrm{Pt}-20 \%$ Rh wire. 
Following the preliminary heat treatment, the disks were ground, using a mullite mortar and pestle, remixed with a few drops of distilled water, and new disks, about $1 / 4$ in. high, were formed at 15,000 $\mathrm{lb} /$ in. $^{2}$ in either a $1 / 2$-in. or $1 / 4$-in.-diam mold. These specimens were then ready to be used for solid-state reaction studies. Some of the specimens used were prepared by G. R. Shelton of the Bureau during the last 10 vears by methods similar to those described here. Most of these specimens were reheated, in the present study, to obtain better crystallinity.

\subsection{Study of Solid-State Reactions}

In the study of solid-state reactions, a conventional platinum-wound quench furnace was used. The temnerature in the furnace was measured with a $\mathrm{Pt}$ versus $\mathrm{Pt}-10$-percent- $\mathrm{Rh}$ thermocouple and controlled by a modified Roberts-type controller. The quenching technique was used because it has been observed that sharper X-ray patterns are often obtained by very fast cooling of the specimen. Phase transitions in the pyrochlore structure are nrobably completely reversible and cannot be "frozen in" by quenching. For quench tests, the $1 / 2^{-}$or $1 / 4-i n$.-diam disks were placed on a platinum platform, which was then suspended in the furnace. The test temperature ranged from $1,250^{\circ}$ to $1,550^{\circ} \mathrm{C}$ and was maintained constant for a given length of time. Equilibrium conditions were usually reached in less than $3 \mathrm{hr}$. Equilibrium was believed to have been reached when X-ray patterns of the specimen showed only a single phase, or when the pattern of a two-phase specimen showed no change with successive heat treatment. After this reaction time, the sample was quenched in air by lifting the specimen disk on its platinum platform out of the hot furnace. The specimen cooled to room temperature in less than $2 \mathrm{~min}$. The samples were then examined by X-ray diffraction, using a Geiger counter diffractometer employing nickel-filtered copper radiation.

In the case of specimens containing $\mathrm{UO}_{2}$, an argon atmosphere was used and the specimens were not quenched. The heating element of the furnace used was a molybdenum-wound stabilized $\mathrm{ZrO}_{2}$ tube and is similar to the one described by Davenport et al. [6].

\section{The Pyrochlore-Structure Type}

The structure of the mineral pyrochlore and of related mineral types has been described by several workers $[3,7,8]$. Pyrochlore has the formula $\mathrm{NaCaNbTaO}_{6} \mathrm{~F}$, in which $\mathrm{Na}$ and $\mathrm{Ca}$ can be considered the $\mathrm{A}$ ions, and $\mathrm{Nb}$ and $\mathrm{Ta}$ the $\mathrm{B}$ ions of an $A_{2} B_{2} X_{7}$ structure. The space group for this cubic compound has been found to be $\mathrm{Fd} 3 \mathrm{~m}-\mathrm{O}_{h}{ }^{7}$, with $Z=8$ and a unit cell of 10.35 to $10.40 \mathrm{~A}$.

In the following description of the pyrochlore structure, the cell origin has been taken at a center of symmetry and the sets of positions referred to may be found on page 341 of reference [9]. In this structure type there are $16 \mathrm{~A}$ ions in position (c), $16 \mathrm{~B}$ ions in position (d), and 8 negative ions in position (a); for the mineral pyrochlore these are the fluorine ions. The remainder of the negative ions (48 oxygen) are in position (f), which contains only one unknown parameter. From a consideration of the spatial requirements in the pyrochlore structure, it is obvious that the value of the unknown parameter must be equal to or very close to $3 / 8$. If the value of $3 / 8$ is used, then all possible $x y z$ combinations of $1 / 8,3 / 8,5 / 8$, and $7 / 8$ are occupied by oxygen, except the eight sites required by the special position (b), which are vacant. In such a structure, if these vacant sites were also occupied, the formula type would be $\mathrm{A}_{2} \mathrm{~B}_{2} \mathrm{O}_{8}$ or $\mathrm{ABO}_{4}$ instead of $\mathrm{A}_{2} \mathrm{~B}_{2} \mathrm{O}_{7}$. The pyrochlore-structure type may therefore be compared to a fluorite structure with 1 out of every 8 negative ions missing. From data given by Bystrom [3], the value of $x$ in the oxygen position for $\mathrm{Cd}_{2} \mathrm{Nb}_{2} \mathrm{O}_{7}$ and $\mathrm{Cd}_{2} \mathrm{Ta}_{2} \mathrm{O}_{7}$ can be taken as 0.414 instead of 0.375 for $3 / 8$. It will be seen in the following discussions that the value of $x$ is probably not the same for all of the pyrochlore-type compounds.

In addition to the extinctions to be expected from a face-centered cell, there are two special extinction rules due to the particular positions of the ions in the pyrochlore-type structure:

(1) The positions (a) and (f), filled by negative ions, are such that possible reflections are limited to the type $h k l$ where $h+k+l=2 n+1$ or $4 n$.

(2) The positions (c) and (d), filled by positive ions, are such that possible reflections are limited to planes of the type

$$
\left.\left.\left.\begin{array}{rl}
h k l: h=2 n+1 \\
k=2 n+1 \\
l=2 n+1
\end{array}\right\} \quad \begin{array}{rr}
4 n+2 \\
\text { or } & 4 n+2 \\
4 n+2
\end{array}\right\} \text { or } \begin{array}{r}
4 n \\
\\
\end{array}\right\}
$$

The result is that, in the pyrochlore-type diffraction patterns, certain reflections are always absent. Of those that may be observed, some are due only to metal atom scattering, some only to oxygen atom scattering, and the remainder to both metal and oxygen scattering. Therefore, the intensities of the peaks in the X-ray powder diffraction patterns are characteristic of the pyrochlore structure, and are evidence for the existence of such a structure. Intensity calculations have been made for some of the cubic compounds studied and will be discussed in later sections.

\section{Results and Discussion}

\subsection{General}

The X-ray diffraction powder patterns prepared in this study revealed that many of the mixtures formed binary compounds of the type $\mathrm{A}_{2} \mathrm{~B}_{2} \mathrm{O}_{7}$. The great majority of the compounds were cubic, essentially isostructural with the mineral pyrochlore. Table 1 lists the indexed X-ray diffraction powder patterns for these cubic compounds. All of the hkl 
TABLE 1. X-ray diffraction powder data for the compounds $\mathrm{Sm}_{2} \mathrm{Ti}_{2} \mathrm{O}_{7}, \mathrm{Gd}_{2} \mathrm{Ti}_{2} \mathrm{O}_{7}, \mathrm{Dy}_{2} \mathrm{Ti}_{2} \mathrm{O}_{7}, \mathrm{Y}_{2} \mathrm{Ti}_{2} \mathrm{O}_{7}, \mathrm{Yb}_{2} \mathrm{Ti}_{2} \mathrm{O}_{7}, \mathrm{La}_{2} \mathrm{Sn}_{2} \mathrm{O}_{7}, \mathrm{Nd}_{2} \mathrm{Sn}_{2} \mathrm{O}_{7}, \mathrm{La}_{2} \mathrm{Zr}_{2} \mathrm{O}_{7}, \mathrm{Nd}_{2} \mathrm{Zr}_{2} \mathrm{O}_{7}$ and the possible compounds $\mathrm{Y}_{2} \mathrm{Zr}_{2} \mathrm{O}_{7}$ and $\mathrm{Nd}_{2} \mathrm{U}_{2} \mathrm{O}_{7}$

\begin{tabular}{|c|c|c|c|c|c|c|c|c|c|c|c|c|c|c|c|c|c|c|c|c|c|c|}
\hline \multirow{2}{*}{$h k l$} & \multicolumn{2}{|c|}{$\mathrm{Sm}_{2} \mathrm{Ti}_{2} \mathrm{O}_{7}$} & \multicolumn{2}{|c|}{$\mathrm{Gd}_{2} \mathrm{Ti}_{2} \mathrm{O}_{7}$} & \multicolumn{2}{|c|}{$\mathrm{Dy}_{2} \mathrm{Ti}_{2} \mathrm{O}_{7}$} & \multicolumn{2}{|c|}{$\mathrm{Y}_{2} \mathrm{Ti}_{2} \mathrm{O}_{7}$} & \multicolumn{2}{|c|}{$\mathrm{Yb}_{2} \mathrm{Ti}_{2} \mathrm{O}_{7}$} & \multicolumn{2}{|c|}{$\mathrm{La}_{2} \mathrm{Sn}_{2} \mathrm{O}_{7}$} & \multicolumn{2}{|c|}{$\mathrm{Nd}_{2} \mathrm{Sn}_{2} \mathrm{O}_{7}$} & \multicolumn{2}{|c|}{$\mathrm{La}_{2} \mathrm{Zr}_{2} \mathrm{O}_{7}$} & \multicolumn{2}{|c|}{$\mathrm{Nd}_{2} \mathrm{Zr}_{2} \mathrm{O}_{7}$} & \multicolumn{2}{|c|}{$\mathrm{Y}_{2} \mathrm{Zr}_{2} \mathrm{O}_{7}(?)$} & \multicolumn{2}{|c|}{$\mathrm{Nd}_{2} \mathrm{U}_{2} \mathrm{O}_{7}(?)$} \\
\hline & $d$ & $I^{\mathrm{a}}$ & $d$ & $I^{\mathrm{a}}$ & $d$ & $I^{\mathrm{a}}$ & $d$ & $I^{\mathrm{a}}$ & $d$ & $I^{\mathrm{a}}$ & $d$ & $I^{\mathrm{a}}$ & $d$ & $I^{\mathrm{a}}$ & $d$ & $I^{\mathrm{a}}$ & $d$ & $I^{\mathrm{a}}$ & $d$ & $I^{\mathrm{a}}$ & $d$ & $I^{\mathrm{a}}$ \\
\hline $\begin{array}{l}111 \\
200 \text { b }\end{array}$ & 5. 91 & 22 & 5. 86 & 18 & 5.80 & 19 & 5. 79 & 22 & 5. 77 & 54 & 6. 18 & 7 & 6. 08 & 4 & 6. 21 & 5 & 6.14 & 5 & $\cdots$ & -- & $\cdots$ & $\ldots$ \\
\hline 220 & 3. 09 & 22 & 3. 06 & 11 & 3. 04 & 15 & 3. 03 & 9 & 3. 02 & 63 & 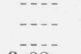 & & 3. 18 & 4 & & $\ldots$ & 3.21 & 4 & $\cdots$ & $-\cdots$ & $\cdots$ & $\cdots$ \\
\hline${ }_{222}^{11}$ & & c $100+$ & $\begin{array}{l}.00 \\
2.94\end{array}$ & $\begin{array}{l}11 \\
100\end{array}$ & $\begin{array}{l}\text { 2. } 94 \\
\text {. } 91\end{array}$ & $\cdot 100+$ & $\begin{array}{l}\text { P. } \\
\text { 2. } 91\end{array}$ & 356 & $\begin{array}{l}\text { D. } \\
\text { 2. } 89\end{array}$ & $\mathrm{c} 100+++$ & 3. 08 & $100+++$ & $\begin{array}{l}\text {. } \\
\text { 3. } 04\end{array}$ & $100+$ & 3. 11 & $100++$ & $\begin{array}{l}3.21 \\
3.07\end{array}$ & $\stackrel{4}{\mathrm{e}} 100+$ & 3.00 & $100++$ & 3. 12 & $100+$ \\
\hline $\begin{array}{l}400 \\
331\end{array}$ & $\begin{array}{l}2.56 \\
2.35\end{array}$ & $\begin{array}{l}60 \\
36\end{array}$ & $\begin{array}{l}\text { 2. } 54 \\
2.33\end{array}$ & $\begin{array}{l}19 \\
16\end{array}$ & $\begin{array}{l}2.52 \\
\text { 2. } 31\end{array}$ & $\begin{array}{l}45 \\
32\end{array}$ & 2. 52 & $\begin{array}{l}45 \\
62\end{array}$ & 2. 50 & $\begin{array}{l}81 \\
78\end{array}$ & $\begin{array}{l}2.67 \\
2.45\end{array}$ & $\begin{array}{r}91 \\
7\end{array}$ & $\begin{array}{l}2.64 \\
2.42\end{array}$ & 77 & $\begin{array}{l}\text { 2. } 69 \\
2.47\end{array}$ & $\begin{array}{l}60 \\
11\end{array}$ & 2. 66 & 48 & 2. 59 & 73 & 2. 71 & 69 \\
\hline $\begin{array}{l}331 \\
420 \mathrm{~b}\end{array}$ & $\begin{array}{l}2.35 \\
2-07-5\end{array}$ & $\cdots$ & .... & 10 & $\begin{array}{l}2.31 \\
--.-\end{array}$ & 32 & 205 & $\begin{array}{c}62 \\
--5\end{array}$ & & 78 & 2. 45 & 7 & & 7 & 2. 47 & 11 & 2. 44 & & -... & $-\cdots$ & -... & $\cdots$ \\
\hline $\begin{array}{c}422 \\
511 / 333\end{array}$ & $\begin{array}{l}\text { 2. } 076 \\
\text { 1. } 969\end{array}$ & $\begin{array}{r}4 \\
14\end{array}$ & 1. 958 & 10 & 1. 943 & 13 & $\begin{array}{l}\text { 2. } 056 \\
\text { 1. } 940\end{array}$ & $\begin{array}{r}5 \\
16\end{array}$ & $\begin{array}{l}\text { 2. } 044 \\
\text { 1. } 929\end{array}$ & $\begin{array}{r}3 \\
39\end{array}$ & 2. 058 & 5 & $\begin{array}{l}\text { 2. } 153 \\
\text { 2. } 030\end{array}$ & $\begin{array}{l}3 \\
3\end{array}$ & 2. $07 \overline{5}$ & 5 & $\begin{array}{l}2.172 \\
2.046\end{array}$ & $\begin{array}{l}3 \\
5\end{array}$ & 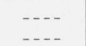 & 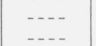 & $\cdots$ & $\cdots$ \\
\hline 440 & 1. 809 & 68 & 1. 799 & 39 & 1. 785 & 75 & 1. 782 & 170 & 1. 772 & c100++ & 1. 809 & $\cdot 100++$ & 1. 866 & 100 & 1. 907 & ${ }^{\mathrm{c}} 100+$ & 1. 882 & 70 & 1. 837 & e100+ & 1. 919 & 72 \\
\hline $\begin{array}{c}531 \\
600 / 442 \text { b }\end{array}$ & $\begin{array}{r}1.730 \\
-.-.\end{array}$ & 11 & $\begin{array}{r}1.720 \\
\ldots\end{array}$ & $\begin{array}{r}8 \\
--.-\end{array}$ & 1. 707 & 10 & & $\begin{array}{r}7 \\
-\end{array}$ & 694 & 42 & $\cdots$ & -.... & 1. 780 & 3 & $\ldots$ & . & 1. 799 & 3 & $\cdots$ & - & $\ldots$ & $\cdots$ \\
\hline $\begin{array}{l}620 \\
533\end{array}$ & $\cdots$ & $\cdots$ & $\cdots$ & $\cdots$ & -... & $\cdots$ & 1. 593 & 3 & 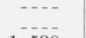 & & -... & & $\cdots$ & - & -... & $\cdots$ & -... & -.. & $\cdots$ & $-\cdots$ & $\cdots$ & $\cdots$ \\
\hline $\begin{array}{l}533 \\
622\end{array}$ & $\begin{array}{l}1.560 \\
1.543\end{array}$ & 6 & $-\cdots$ & $\cdots$ & 190 & $\cdots$ & & & 1. 539 & 8 & $\cdots$ & -..... & $\ldots$ & $\cdots$ & $\cdots$ & $-\cdots$ & 1. 632 & 4 & --.- & -..- & $--\cdot$ & $\cdots$ \\
\hline $\begin{array}{l}622 \\
444 \\
\end{array}$ & $\begin{array}{l}\text { 1. } 543 \\
\text { 1. } 477\end{array}$ & $\begin{array}{l}41 \\
17\end{array}$ & $\begin{array}{l}\text { 1. } 535 \\
\text { 1. } 469\end{array}$ & $\begin{array}{l}31 \\
10\end{array}$ & $\begin{array}{l}\text { 1. } 522 \\
\text { 1. } 458\end{array}$ & $\begin{array}{l}58 \\
13\end{array}$ & $\begin{array}{l}\text { 1. } 520 \\
\text { 1. } 456\end{array}$ & $\begin{array}{l}56 \\
13\end{array}$ & $\begin{array}{l}\text { 1. } 511 \\
\text { 1. } 447\end{array}$ & $\begin{array}{c}{ }^{c} 100+ \\
57\end{array}$ & $\begin{array}{l}\text { 1. } 613 \\
\text { 1. } 544\end{array}$ & ${ }_{29}^{\mathrm{c}} 100+$ & $\begin{array}{l}\text { 1. } 592 \\
\text { 1. } 524\end{array}$ & $\begin{array}{l}84 \\
25\end{array}$ & $\begin{array}{l}\text { 1. } 626 \\
\text { 1. } 557\end{array}$ & $\begin{array}{l}88 \\
22\end{array}$ & $\begin{array}{l}\text { 1. } 605 \\
\text { 1. } 537\end{array}$ & $\begin{array}{l}63 \\
16\end{array}$ & 1. 567 & $\begin{array}{l}73 \\
14\end{array}$ & 1. 638 & $\begin{array}{l}69 \\
16\end{array}$ \\
\hline $\begin{array}{r}711 / 551 \\
640 \mathrm{~b}\end{array}$ & 1. 432 & 6 & 1. 425 & 3 & 1. 415 & 5 & 1. 412 & 4 & 1. 404 & 14 & $1.0+4$ & & 1,024 & $-\ldots$ & 1.004 & 22 & 1. 491 & $\begin{array}{r}10 \\
3\end{array}$ & & & & \\
\hline $\begin{array}{l}640 \mathrm{~b} \\
642\end{array}$ & -... & -.- & $\cdots$ & $\cdots$ & $\cdots$ &.-- & -..- & $\cdots$ & $\cdots$ & $\cdots$ & $-\cdots$ & $\cdots$ & -... & $\cdots$ & -... & -.... & --.- & $-\cdots$ & $-\cdots$ & $-\cdots$ & -... & $\cdots$ \\
\hline $731 / 553$ & 1. 332 & 8 & 1. 325 & 3 & 1. 316 & 5 & 1. 313 & 5 & 1. 305 & 14 & & & & & & & 1. 386 & 2 & & & & \\
\hline $\begin{array}{l}800 \\
733\end{array}$ & & 9 & 1. 273 & 5 & & 10 & 1. 261 & 10 & 1. 254 & 15 & 1. 337 & 15 & 1. 320 & 17 & 1. 349 & 13 & 1. 331 & 11 & 1. 300 & 11 & 1. 360 & 14 \\
\hline $820 / 644$ b & 1. 250 & $\begin{array}{r}4 \\
---\end{array}$ & 1. 242 & 3 & 1. 234 & $\begin{array}{r}5 \\
---\end{array}$ & 1. 233 & 5 & 1. 225 & 8 & 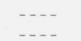 & & 1. 288 & 3 & 1. 319 & 3 & 1. 296 & 3 & $\cdots$ & .... & $\cdots$ & $\ldots$ \\
\hline $822 / 660$ & --- & -.. & 1. 202 & 3 & -..- & $\cdots$ & 1. 190 & 3 & 1. 181 & 3 & $\ldots$ & -.. & & -... & -...- & $\cdots$ & $\cdots$ & $\cdots$ & -..- & -... & $\cdots$ & $\cdots$ \\
\hline $751 / 555$ & 1. 182 & $\begin{array}{r}6 \\
19\end{array}$ & 168 & 111 & 1. 167 & 5 & 1157 & 85 & 1. 158 & $\begin{array}{l}10 \\
45\end{array}$ & 1.227 & 37 & 1212 & 30 & 1. 245 & $\begin{array}{r}3 \\
38\end{array}$ & 1. 229 & $\begin{array}{r}4 \\
24\end{array}$ & 192 & 18 & 249 & \\
\hline $\begin{array}{l}602 \\
840\end{array}$ & $\begin{array}{l}144 \\
144\end{array}$ & $\begin{array}{l}19 \\
19\end{array}$ & $\begin{array}{l}\text { 1. } 108 \\
\text { 1. } 138\end{array}$ & $\begin{array}{r}11 \\
9\end{array}$ & $\begin{array}{l}\text { 1. } 159 \\
\text { 1. } 130\end{array}$ & $\begin{array}{l}19 \\
13\end{array}$ & $\begin{array}{l}\text { 1. } 128 \\
\text { 1. } 128\end{array}$ & $\begin{array}{l}80 \\
17\end{array}$ & $\begin{array}{l}\text { 1. } 1 . \\
1.1\end{array}$ & $\begin{array}{l}45 \\
37\end{array}$ & 1. 227 & $\begin{array}{l}31 \\
29\end{array}$ & 1. 181 & 焉 & 1. 207 & 夏28 & $\begin{array}{l}1.22 \\
1.19\end{array}$ & $\begin{array}{l}24 \\
16\end{array}$ & $\begin{array}{l}1.192 \\
1.162\end{array}$ & $\begin{array}{l}18 \\
11\end{array}$ & $\begin{array}{l}\text { 1. } 249 \\
\text { 1. } 217\end{array}$ & 20 \\
\hline $\begin{array}{r}911 / 753 \\
842 \mathrm{~b}\end{array}$ & 1. 123 & 8 & 1. 117 & 4 & 1. 109 & 5 & 1. 108 & 3 & 1. 101 & 15 & $\cdots$ & & $\ldots$ & 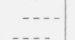 & $\cdots$ & -..- & 1. 169 & 3 & 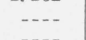 & -- & & $\ldots$ \\
\hline 664 & & $=$ & & $\ldots$ & -... & -.. & -.. & ... & & & & & & $\cdots$ & - & .... & $\ldots$ & -... & -... & -... & -... & $\cdots$ \\
\hline $\begin{array}{l}931 \\
844\end{array}$ & & $\begin{array}{r}5 \\
15\end{array}$ & 1. 039 & 8 & 1031 & 12 & 1030 & 51 & 1. 051 & $\begin{array}{r}3 \\
25\end{array}$ & 1092 & 26 & 1078 & 22 & 102 & $17--$ & 1087 & 17 & 1062 & 16 & $\cdots$ & 17 \\
\hline $\begin{array}{l}933 / 771 / 755 \\
10,0,0 / 860 \mathrm{~b}\end{array}$ & 1. 028 & 7 & 1. 023 & 4 & 1. 016 & 5 & 1. 014 & 5 & 1. 008 & $\begin{array}{r}25 \\
8\end{array}$ & & 20 & & 22 & 1. 102 & & $\begin{array}{l}1.087 \\
1.067\end{array}$ & $\begin{array}{r}17 \\
3\end{array}$ & 1.062 & 16 & 1.111 & 17 \\
\hline $10,2,0 / 862$ & & 5 & -... & $\ldots$ & .... & ... & & & & & $\cdots$ & 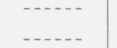 & $\ldots$ & $\ldots$ & $\ldots$ & $\ldots$ & $\ldots$ & $\ldots$ & $\cdots$ & $-\cdots-$ & $\cdots$ & $\cdots$ \\
\hline $\begin{array}{c}951 / 773 \\
10,2,2 / 666\end{array}$ & $\begin{array}{l}0.9895 \\
.9841\end{array}$ & $\begin{array}{r}5 \\
16\end{array}$ & 0.9797 & 9 & 0.9726 & 13 & $\begin{array}{r}0.9757 \\
.9711\end{array}$ & $\begin{array}{r}3 \\
12\end{array}$ & $\begin{array}{r}0.9693 \\
.9646\end{array}$ & $\begin{array}{r}4 \\
23\end{array}$ & 1. 030 & 28 & 1. 017 & 22 & 1. 039 & 20 & 1. 025 & 15 & 1.001 & 12 & 1. 048 & 19 \\
\hline $\begin{array}{c}953 \\
10,4,0 / 864 \text { b }\end{array}$ & --- & $-\cdots$ & $-\cdots$ & $-\cdots$ & $\cdots$ & $--\cdot$ & $-\cdots$ & -... & .9352 & 3 & $-\cdots$ & & $\cdots$ & $\cdots$ & $\ldots$ & -... & $\ldots$ & $-\cdots$ & -... & -... & -... & -..- \\
\hline $10,4,2$ & $-\cdots$ & -.. & - & $\ldots$ & - &.- & $\cdots$ & - & -... & & .... & $\cdots$ & .... & $\ldots$ & $\ldots$ & $\cdots$ & .... & $\cdots$ & -... & $-\cdots$ & -... & $\cdots$ \\
\hline $11, \frac{1,1 / 775}{880}$ & 9041 & 13 & 9000 & 5 & 893 & $--\overline{7}$ & 8926 & 4 & .9043 & $\begin{array}{r}4 \\
12\end{array}$ & 0.450 & 11 & 0.9340 & 8 & 0050 & 7 & $00-11$ & 6 & 00109 & (n) & 00098 & $\begin{array}{ll}0 \\
-1\end{array}$ \\
\hline $11,3,1 / 971 / 955$ & .8938 & 6 & 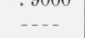 & 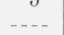 & .8829 & 5 & .8819 & ${ }_{3}^{+}$ & .8000 & 8 & $0.9+59$ & & $0.95+0$ & $\ldots$ & 0.9540 & $\ldots$ & $\begin{array}{l}0.9411 \\
.9304\end{array}$ & $\begin{array}{l}0 \\
5 \\
5\end{array}$ & $\begin{array}{l}0.9192 \\
-. .-\end{array}$ & 5 & 0.9628 & 8 \\
\hline & $-\cdots$ & $\cdots$ & $\cdots-$ & $\cdots$ & $\cdots$ & $\cdots$ & $\cdots$ & -... & $\cdots$ & -... & $-\cdots$ & $-\cdots$ & $\cdots$ & $\cdots$ & $\cdots-$ & -- & -..- & $\cdots-\cdot$ & --. & $\cdots$ & $\cdots$ & -.- \\
\hline & .8676 & 5 & & & & & & & 8507 & & & & & & & & & & & & & \\
\hline & & 13 & .860 & 13 & .8541 & 15 & .8530 & 12 & & 28 & .9045 & 30 & 89 & 26 & .912 & 23 & .900 & 18 & .879 & 11 & .9208 & 19 \\
\hline & & 17 & .8456 & 8 & .8421 & 12 & .8410 & 35 & .8359 & 21 & .8918 & 21 & .8806 & 18 & .8995 & 14 & .8874 & $\begin{array}{l}10 \\
13\end{array}$ & .8667 & 7 & .9078 & 12 \\
\hline & .8438 & 5 &..- & -... & -... & $\ldots$ & .... & $\ldots$ & .8273 & 5 & & & & & 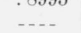 & & .0014 & & .000 & $\ldots$ & .000 & 10 \\
\hline 12 & -..- & $\cdots$ & -... & -... & -..- & -- & -.- & -..- & -.- & & $\cdots$ & & -..- & & -... & 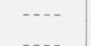 & -..- & $\cdots-$ & --- & $\cdots$ & -.- & $\cdots$ \\
\hline $\begin{array}{r}12,2,2 / 10 \\
11,5,3 / 4\end{array}$ & .8215 & $-\overline{6}$ & .817 & 4 & .81 & 5 & .81 & 5 & .8057 & -5 & $\cdots$ & & $\cdots$ & $\cdots$ & $\cdots$ & --- & $\cdots$ & $\cdots$ & $\cdots$ & $\cdots$ & $-\cdots$ & $\cdots$ \\
\hline 12,4 , & .8085 & 10 & & 7 & .7989 & 7 & .7980 & 7 & .7930 & 15 & .8461 & 18 & .8355 & 15 & .8533 & 11 & .8418 & 12 & .8224 & 7 & .8615 & 11 \\
\hline $\begin{array}{c}991 \\
12,4,2 / 10,8,0 / 886 \text { b }\end{array}$ & --.- & $\cdots$ & -... & $-\cdots$ & --- &.-- & $-\cdots$ & $\cdots$ & $-\cdots$ & & $\cdots$ & $\cdots$ & $\cdots$ & -... & $\cdots$ & $\cdots$ & $-\cdots$ & $\cdots$ & $\ldots$ & $\cdots$ & $\cdots$ & $\cdots$ \\
\hline $10,8,2$ & & & $\ldots$ & $\ldots$ & $\ldots$ & ... & .... & -...- & $\ldots$ & .... & $\ldots$ & ... & $\ldots$ & -... & .... & $\ldots$ & .... & $\ldots$ & -... & -...- & .... & .... \\
\hline $13,1,1 / 11,7,1 / 1$ & .7819 & $\begin{array}{r}5 \\
10\end{array}$ & 7787 & 4 & $\cdots$ & -.. & $\cdots$ & $\cdots$ & $\cdots$ & $\cdots+\cdots$ & 8161 & 17 & 8059 & 14 & -1230 & $\cdots$ & sila & $\cdots$ & 3020 & $\cdots$ & . & - \\
\hline & -... & ... & .... & $\ldots$ & $\cdots$ & $\cdots$ & -... & $\cdots$ & $\cdots$ & ........ & .8067 & $\begin{array}{l}18 \\
18\end{array}$ & .7966 & 15 & .8136 & 11 & .8026 & 7 & 7841 & $\begin{array}{l}5 \\
3\end{array}$ & .8216 & 8 \\
\hline $13,3,1 / 11,7,3 / 977$ & $\cdots$ & --- &.-- & -..- & $\cdots$ & ... & -..- & -..- & $\cdots$ & $\cdots$ & -... & & .... & .... & -... & & -..- & $\ldots$ & -... & & -... & \\
\hline $12,6,0$ & -... & ... & .... & .... & $\ldots .$. & ...- & -... & $\ldots$ & .... & ...... & .... & $\ldots$ & $\ldots . .$. & .... & .... & .... & .... & .... & .... & $\ldots$ & $\ldots$ & $\ldots$ \\
\hline & $\cdots$ & $\cdots$ & $\cdots$ & $\cdots$ & $\cdots$ & $\cdots$ & $\cdots$ & - & $-\cdots$ & & $\cdots$ & -...-. & $\cdots-\cdot$ & -... & $\cdots$ & $\cdots$ & 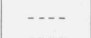 & $\cdots-$ & $\cdots$ & -..- & $\cdots$ & $\cdots$ \\
\hline 888 & $\ldots$ & $\ldots$ & -... & .... & .... & $\cdots$ & .... & $\ldots$ & .... & 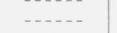 & $\ldots$ & $\ldots$ & $\cdots$ & ... & .7788 & 5 & 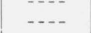 & $\cdots$ & .... & .... & .7865 & 5 \\
\hline
\end{tabular}

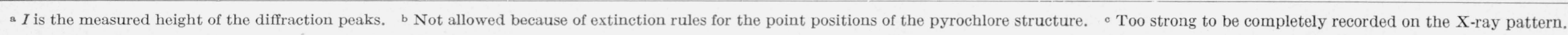


TABLE 2. Heat treatment and structure type of compositions studied

\begin{tabular}{|c|c|c|c|c|c|c|c|}
\hline \multirow{2}{*}{ Composition } & \multirow{2}{*}{$\underset{\text { of } \mathrm{A}^{+3}}{\text { Radius a }}$} & \multirow{2}{*}{$\underset{\text { of } \mathrm{B}^{+4}}{\text { Radius a }}$} & \multicolumn{2}{|c|}{ Heat treatment b } & \multirow{2}{*}{ Structure type } & \multirow{2}{*}{ Symmetry } & \multirow{2}{*}{ Parameter } \\
\hline & & & $\begin{array}{c}\text { Temper- } \\
\text { ature }\end{array}$ & Time & & & \\
\hline $\begin{array}{l}\mathrm{La}_{2} \mathrm{Ti}_{2} \mathrm{O}_{7} \\
\mathrm{Nd}_{2} \mathrm{Ti}_{2} \mathrm{O}_{7} \\
\mathrm{Sn}_{2} \mathrm{Ti}_{2} \mathrm{O}_{7} \\
\mathrm{Gd}_{2} \mathrm{Ti}_{2} \mathrm{O}_{7} \\
\mathrm{Dy}_{2} \mathrm{Ti}_{2} \mathrm{O}_{7}\end{array}$ & $\begin{array}{l}1.14 \\
1.04 \\
1.00 \\
0.97 \\
.92\end{array}$ & $\begin{array}{r}0.68 \\
.68 \\
.68 \\
.68 \\
.68\end{array}$ & $\begin{array}{l}{ }^{\circ} C \\
1,550 \\
1,550 \\
1,550 \\
1,550 \\
1,425\end{array}$ & $\begin{array}{r}h r \\
1 \\
1 \\
1 / 2 \\
2 \\
1\end{array}$ & $\begin{array}{l}\begin{array}{l}\text { Distorted pyrochlore } \\
\text { do } \\
\text { Pyrochlore }\end{array} \\
\text { do }\end{array}$ & $\begin{array}{l}\text { Unknown } \\
\text { Cubic } \\
\text { do }\end{array}$ & $\begin{array}{l}10.228 \\
10.181 \\
10.106\end{array}$ \\
\hline $\begin{array}{l}\mathrm{Y}_{2} \mathrm{Ti}_{2} \mathrm{O}_{7} \\
\mathrm{Yb}_{2} \mathrm{Ti}_{2} \mathrm{O}_{7} \\
\mathrm{In}_{2} \mathrm{O}_{3}: 2 \mathrm{TiO}_{2} \\
\mathrm{Sb}_{2} \mathrm{O}_{3}: 2 \mathrm{TiO}_{2} \\
\mathrm{La}_{2} \mathrm{Sn}_{2} \mathrm{O}_{7}\end{array}$ & $\begin{array}{r}.92 \\
.86 \\
.81 \\
.76 \\
1.14\end{array}$ & $\begin{array}{l}.68 \\
.68 \\
.68 \\
.68 \\
.71\end{array}$ & $\begin{array}{l}1,525 \\
1,550 \\
1,550 \\
1,475 \\
1,550\end{array}$ & $\begin{array}{r}1 / 2 \\
2 \\
3 \\
2 \\
1\end{array}$ & $\begin{array}{l}\text { No compound } \\
\text { Pyrochlore } \\
\text { do }\end{array}$ & _... do & $\begin{array}{l}10.093 \\
10.030 \\
-\overline{10.702}\end{array}$ \\
\hline $\begin{array}{l}\mathrm{Nd}_{2} \mathrm{Sn}_{2} \mathrm{O}_{7} \\
\mathrm{Bi}_{2} \mathrm{Sn}_{2} \mathrm{O}_{7} \\
\mathrm{La}_{2} \mathrm{Zr}_{2} \mathrm{O}_{7} \\
\mathrm{Nd}_{2} \mathrm{Zr}_{2} \mathrm{O}_{7} \\
\mathrm{Y}_{2} \mathrm{Zr}_{2} \mathrm{O}_{7}\end{array}$ & $\begin{array}{l}\text { 1. } 04 \\
0.96 \\
\text { 1. } 14 \\
\text { 1. } 04 \\
0.92\end{array}$ & $\begin{array}{l}.71 \\
.71 \\
.79 \\
.79 \\
.79\end{array}$ & $\begin{array}{l}1,550 \\
1,250 \\
1,550 \\
1,550 \\
1,550\end{array}$ & $\begin{array}{l}1 \\
1 \\
3 \\
2 \\
2\end{array}$ & $\begin{array}{l}\text { Disto } \\
\text { Pyrochlod pyrochlore } \\
\text { do }\end{array}$ & $\begin{array}{l}\text { Unknown do } \\
\text { Cubic_-_a }\end{array}$ & $\begin{array}{l}10.568 \\
10.793 \\
10.648 \\
10.402(?)\end{array}$ \\
\hline $\begin{array}{l}\mathrm{In}_{2} \mathrm{O}_{3}: 2 \mathrm{ZrO}_{2} \\
\mathrm{In}_{2} \mathrm{O}_{3}: 2 \mathrm{CeO}_{2} \\
\mathrm{Nd}_{2} \mathrm{O}_{3}: 2 \mathrm{UO}_{2} \\
\mathrm{Y}_{2} \mathrm{O}_{3}: 2 \mathrm{UO}_{2}\end{array}$ & $\begin{array}{r}.81 \\
.81 \\
1.04 \\
0.92\end{array}$ & $\begin{array}{l}.79 \\
.94 \\
.97 \\
.97\end{array}$ & $\begin{array}{r}1,550 \\
1,350 \\
\circ 1,600 \\
\cdot 1,600\end{array}$ & $\begin{array}{r}3 \\
1 / 2 \\
1 \\
1\end{array}$ & $\begin{array}{l}\text { No compound } \\
\text { Fluorite solid solution (?) } \\
\text { No compound }\end{array}$ & Cubic & 5.449 \\
\hline
\end{tabular}

a Radius of the ions taken from Green [10].

b All specimens, unless otherwise stated, were quenched from the designated temperature.

- These specimens were heated in an argon atmosphere to prevent the oxidation of $\mathrm{UO}_{2}$ and were not quenched.

values allowed by a face-centered lattice have been listed, up to and including $h^{2}+k^{2}+l^{2}=192$. It may be seen that none of the peaks forbidden by the structural arrangement of the pyrochlore type are observed in the X-ray patterns of any of the compounds studied.

Table 2 lists the lattice parameters of these compounds, together with the composition, heat treatment, and structure identification for all the materials examined. All compounds and mixtures studied have been plotted in figure 1 on the basis of constituent ionic radii. The values for the radii of the ions have been taken from Green's Geochemical Table of the Elements [10]. The radius of the $\mathrm{A}^{+3}$ ion is plotted as the ordinate and that of the $\mathrm{B}^{+4}$ ion as the abscissa. It can be seen that only the larger $\mathrm{A}$ ions with the smaller B ions (the upper left of the

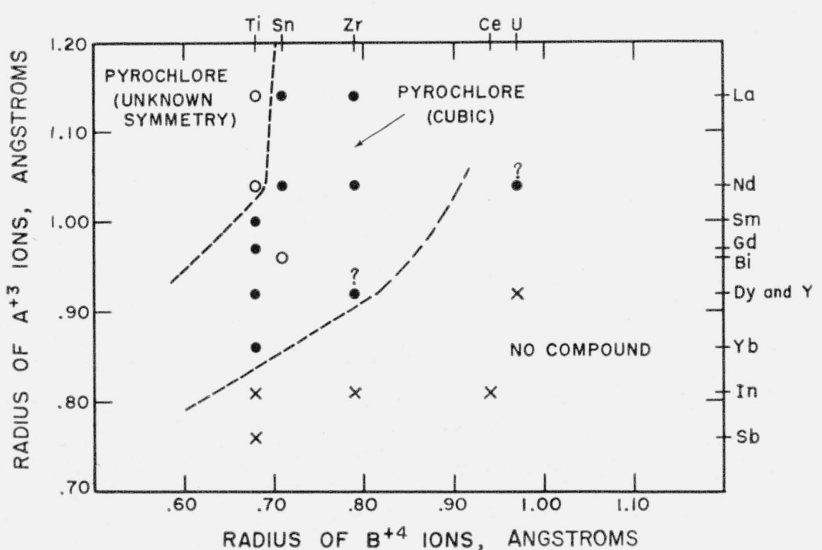

Figure 1. Classification of $\mathrm{A}^{+3}{ }_{2} \mathrm{~B}^{+4}{ }_{2} \mathrm{O}_{7}$ compounds according to the constituent ionic radii.

Pyrochlore (unknown symmetry).

Pyrochlore (cubic).

? Single-phase face-centered cubic.

No compound. diagram) form compounds. In the lower-right portion of the diagram no compounds are indicated. Thus, areas of compound formation and also of symmetry types can be located on this diagram. This is similar to the types of diagrams given by Wood [11] and by Keith and Roy [12] for perovskite structures. Detailed discussion of each of the compounds studied is given in the following sections.

\subsection{Titanates}

Most of the rare-earth titanates form compounds having the cubic pyrochlore structure; however, $\mathrm{La}_{2} \mathrm{Ti}_{2} \mathrm{O}_{7}$ and $\mathrm{Nd}_{2} \mathrm{Ti}_{2} \mathrm{O}_{7}$ are not cubic. The X-ray diffraction powder data for these two compounds are listed in table 3. The X-ray pattern for $\mathrm{La}_{2} \mathrm{Ti}_{2} \mathrm{O}_{7}$ is very sharp and clear; however, that of $\mathrm{Nd}_{2} \mathrm{Ti}_{2} \mathrm{O}_{7}$ is rather diffuse. These compounds seem to be approximately isostructural with $\mathrm{CaZr}^{\top} \mathrm{Ti}_{2} \mathrm{O}_{7}$ [4]. The X-ray patterns of these compounds all have diffraction peaks more or less grouped around the positions that would be expected for a cubic pyrochlore structure. An effort has been made to correlate the structure of these titanates with the orthorhombic form of the $A_{2} B_{2} X_{7}$ compounds exemplified by the mineral weberite, as described by Bystrom [7] for $\mathrm{Ca}_{2} \mathrm{Sb}_{2} \mathrm{O}_{7}$ and other antimonates. The titanates reported in the present study do not seem to have the same structure as that reported for the compounds studied by Bystrom, and the X-ray patterns strongly suggest distortions of the pyrochlore type.

The other four rare-earth titanates studied here have the cubic pyrochlore structure, as does yttrium titanate (table 1). These compounds all give very sharp X-ray patterns, with relatively strong additional lines for the $h k l$ peaks required by the pyrochlore structure as compared to the fluorite structure. 
TABLE 3. X-ray diffraction powder data for the compounds $\mathrm{La}_{2} \mathrm{O}_{3} \cdot 2 \mathrm{TiO}_{2}$ and $\mathrm{Nd}_{2} \mathrm{O}_{3} \cdot 2 \mathrm{TiO}_{2}$

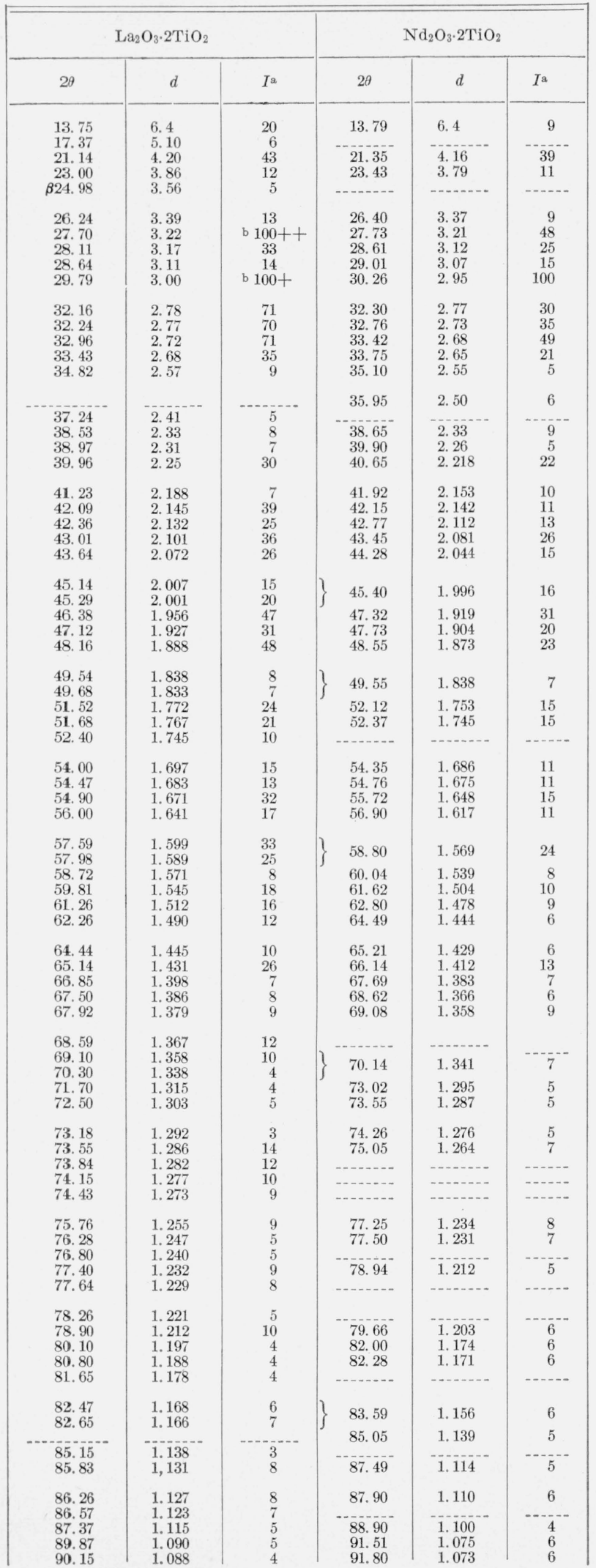

TABLE 3. X-ray diffraction powder duta for the compounds $\mathrm{La}_{2} \mathrm{O}_{3} \cdot 2 \mathrm{TiO}_{2}$ and $\mathrm{Nd}_{2} \mathrm{O}_{3} \cdot 2 \mathrm{TiO}_{2}$ - Continued

\begin{tabular}{|c|c|c|c|c|c|}
\hline \multicolumn{3}{|c|}{$\mathrm{La}_{2} \mathrm{O}_{3} \cdot 2 \mathrm{TiO}_{2}$} & \multicolumn{3}{|c|}{$\mathrm{Nd}_{2} \mathrm{O}_{3} \cdot 2 \mathrm{TiO}_{2}$} \\
\hline $2 \theta$ & $d$ & $I^{\mathrm{a}}$ & $2 \theta$ & $d$ & $I^{\mathrm{a}}$ \\
\hline 91.25 & 1.078 & 5 & _. . . & - & ...... \\
\hline 91.57 & 1. 075 & 7 & - & - & - n \\
\hline 92.50 & 1. 066 & 9 & 94.06 & 1.053 & 6 \\
\hline 93.69 & 1. 056 & 13 & 95.57 & 1. 040 & 7 \\
\hline - & - & - & 96.72 & & \\
\hline 95.13 & 1. 044 & 5 & 97.20 & 1. 027 & 7 \\
\hline 96.15 & 1. 035 & 7 & 97.82 & 1.022 & 6 \\
\hline $\begin{array}{l}98.04 \\
98.98\end{array}$ & $\begin{array}{l}1.020 \\
1.013\end{array}$ & $\begin{array}{l}4 \\
3\end{array}$ & 100.70 & 1.000 & $-\frac{-1}{5}$ \\
\hline 100.45 & 1. 002 & 6 & 103.12 & 0.9834 & 5 \\
\hline 100.83 & 0.9995 & 5 & 106.02 & .9643 & 4 \\
\hline 103.96 & .9777 & 5 & $\begin{array}{l}100.02 \\
106.60\end{array}$ & $\begin{array}{l}.9043 \\
.9607\end{array}$ & $\frac{4}{7}$ \\
\hline 104. 88 & .9717 & $\begin{array}{l}3 \\
4\end{array}$ & 107.70 & .9539 & 5 \\
\hline $\begin{array}{l}105.22 \\
108.40\end{array}$ & $\begin{array}{l}.9694 \\
.9497\end{array}$ & $\begin{array}{l}4 \\
4\end{array}$ & 110.79 & .9358 & 4 \\
\hline 110.25 & .9388 & 9 & 112.13 & .9284 & 5 \\
\hline 111. 93 & .9294 & 6 & & $--1-1$ & \\
\hline 113.31 & .9221 & 7 & 116.45 & .9061 & 7 \\
\hline 113.84 & .9192 & 8 & 116.87 & .9040 & 2 \\
\hline - & - & ......... & 119.03 & .8938 & \\
\hline & & & 120.40 & .8876 & 6 \\
\hline 120.70 & 8863 & 7 & 124.02 & .8723 & 6 \\
\hline 123.15 & 8758 & 4 & - & - & -.-... \\
\hline 124.08 & .8720 & 4 & - - & 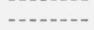 & -.... \\
\hline 124.68 & .8696 & 4 & - & - & ...... \\
\hline 125.32 & .8671 & 4 & ........ & - n & - \\
\hline $\begin{array}{l}128.02 \\
128.35\end{array}$ & .8557 & 3 & - & - & $\ldots$ \\
\hline 128.81 & .8540 & 3 & ......... & ....... & - - \\
\hline 130.14 & .8494 & 5 & - non & - non & - n \\
\hline 130.82 & 8471 & 5 & - & - & ...... \\
\hline 133.35 & .8388 & 8 & 139.41 & .8212 & 6 \\
\hline $\begin{array}{l}130.05 \\
135.10\end{array}$ & $\begin{array}{l}.8300 \\
.8334\end{array}$ & 5 & 108. & 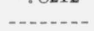 & -... \\
\hline 135.80 & .8313 & 6 & $\ldots$ & - & - n- \\
\hline 137.80 & .8256 & 4 & 149.87 & .7976 & 5 \\
\hline 147.10 & .8031 & 6 & -....... & -...... & $-\ldots$ \\
\hline 148.02 & .8012 & 5 & - n & - & ...... \\
\hline 149.92 & .7976 & 5 & ..... & - & $-\ldots$ \\
\hline 150.45 & .7966 & 8 & 158.45 & .7841 & 6 \\
\hline 151.65 & .7944 & 7 & ....... & - & -..... \\
\hline 152.90 & .7923 & 5 & - n & - & \\
\hline
\end{tabular}

a $I$ is the observed height of the diffraction peaks.

b These peaks were too strong to be completely recorded on the X-ray pattern.

Table 4 shows the intensity values calculated for a few representative planes of $\mathrm{Sm}_{2} \mathrm{Ti}_{2} \mathrm{O}_{7}$ and other compounds. The intensity valves were calculated, using the formula

$$
I_{\text {cale. }} \approx F^{2} \rho \frac{1+\cos ^{2} 2 \theta}{\sin ^{2} \theta \cdot \cos \theta},
$$

where

$F=$ the structure amplitude as given in reference [9, p. 519].

$\rho=$ multiplicity factor, and the balance of the equation refers to the polarization and Lorenz factors for powder patterns.

These intensities were then recalculated on the basis of 100 for the strongest peak. Table 4 shows the individual contribution of the ions in the structure and the final intensity valves compared with the measured peak heights. It can be seen that both the 0.375 value and the 0.414 value of the $x$ parameter for 48 oxygen give poor agreement between observed peak heights and calculated intensity for $\mathrm{Sm}_{2} \mathrm{Ti}_{2} \mathrm{O}_{7}$ although the agreement of the zirconates and stannates is fair for 0.414 . It must be concluded that the oxygen parameter for the titanate compounds is slightly different than that reported by Bystrom [3] for the niobates and tantalates. 
TABLE 4. Calculated versus observed intensities for some of the diffraction peaks of typical $\mathrm{A}_{2}{ }^{+3} \mathrm{~B}_{2}{ }^{+4} \mathrm{O}_{7}$ compounds

\begin{tabular}{|c|c|c|c|c|c|c|c|c|}
\hline \multirow{2}{*}{$h k l$} & \multirow{2}{*}{$\underset{\mathrm{A}^{+3}}{f \times 16}$} & \multirow{2}{*}{$\underset{\mathrm{B}+4}{f \times 16}$} & \multirow{2}{*}{$f \times 8 \mathrm{O}$} & \multicolumn{2}{|c|}{$f \times 480$} & \multicolumn{2}{|c|}{ Calculated $I$ a } & \multirow{2}{*}{$\underset{I \text { a }}{\text { Observed }}$} \\
\hline & & & & $x=0.375$ & $x=0.414$ & $x=0.375$ & $x=0.414$ & \\
\hline \multicolumn{9}{|c|}{$\mathrm{Sm}_{2} \mathrm{Ti}_{2} \mathrm{O}_{7}$} \\
\hline $\begin{array}{c}111 \\
222 \\
400 \\
331 \\
511 / 333\end{array}$ & $\begin{array}{r}-445 \\
829 \\
805 \\
392 \\
-720\end{array}$ & $\begin{array}{r}126 \\
242 \\
232 \\
-112 \\
589\end{array}$ & \begin{tabular}{|r|}
-48 \\
0 \\
-22 \\
30 \\
52
\end{tabular} & $\begin{array}{r}0 \\
0 \\
-269 \\
0 \\
0\end{array}$ & $\begin{array}{r}70 \\
0 \\
-150 \\
66 \\
31\end{array}$ & $\begin{array}{r}50.6 \\
100.0 \\
26.1 \\
9.6 \\
0.9\end{array}$ & $\begin{array}{r}33.1 \\
100.0 \\
35.1 \\
21.7 \\
0.3\end{array}$ & $\begin{array}{c}22 \\
\text { b } 100+ \\
60 \\
36 \\
14\end{array}$ \\
\hline \multicolumn{9}{|c|}{$\mathrm{Nd}_{2} \mathrm{Zr}_{2} \mathrm{O}_{7}$} \\
\hline $\begin{array}{c}111 \\
222 \\
400 \\
331 \\
511 / 333\end{array}$ & $\begin{array}{r}-432 \\
814 \\
786 \\
384 \\
-725\end{array}$ & $\begin{array}{r}273 \\
514 \\
501 \\
-245 \\
458\end{array}$ & $\begin{array}{r}-48 \\
0 \\
-23 \\
31 \\
53\end{array}$ & $\begin{array}{r}0 \\
0 \\
-274 \\
0 \\
0\end{array}$ & $\begin{array}{r}70 \\
0 \\
-153 \\
68 \\
31\end{array}$ & $\begin{array}{r}10.5 \\
100.0 \\
26.7 \\
1.2 \\
4.1\end{array}$ & $\begin{array}{r}4.6 \\
100.0 \\
33.6 \\
5.8 \\
3.0\end{array}$ & $\begin{array}{c}5 \\
\text { b } 100+ \\
48 \\
8 \\
5\end{array}$ \\
\hline \multicolumn{9}{|c|}{$\mathrm{Y}_{2} \mathrm{Zr}_{2} \mathrm{O}_{7}$} \\
\hline $\begin{array}{c}111 \\
222 \\
400 \\
331 \\
511 / 333\end{array}$ & $\begin{array}{r}-271 \\
499 \\
482 \\
238 \\
-442\end{array}$ & $\begin{array}{r}274 \\
512 \\
496 \\
-246 \\
454\end{array}$ & $\begin{array}{r}-48 \\
0 \\
-23 \\
31 \\
53\end{array}$ & $\begin{array}{r}0 \\
0 \\
-274 \\
0 \\
0\end{array}$ & $\begin{array}{r}70 \\
0 \\
-153 \\
68 \\
31\end{array}$ & $\begin{array}{r}0.9 \\
100.0 \\
24.6 \\
0.1 \\
.2\end{array}$ & $\begin{array}{r}0.3 \\
100.0 \\
34.1 \\
1.5 \\
0.8\end{array}$ & $\begin{array}{c}0 \\
\mathrm{~b} 100+ \\
73 \\
0 \\
0\end{array}$ \\
\hline \multicolumn{9}{|c|}{$\mathrm{Nd}_{2} \mathrm{Sn}_{2} \mathrm{O}_{7}$} \\
\hline $\begin{array}{c}111 \\
222 \\
400 \\
331 \\
511 / 333\end{array}$ & $\begin{array}{r}-432 \\
811 \\
782 \\
382 \\
-720\end{array}$ & $\begin{array}{r}350 \\
661 \\
642 \\
-313 \\
589\end{array}$ & $\begin{array}{r}-48 \\
0 \\
-23 \\
31 \\
53\end{array}$ & $\begin{array}{r}0 \\
0 \\
-274 \\
0 \\
0\end{array}$ & $\begin{array}{r}70 \\
0 \\
-153 \\
68 \\
31\end{array}$ & $\begin{array}{r}3.3 \\
100.0 \\
31.5 \\
0.8 \\
.4\end{array}$ & $\begin{array}{r}0.9 \\
100.0 \\
38.6 \\
2.2 \\
0.2\end{array}$ & b $\begin{array}{c}4 \\
100+ \\
77 \\
7 \\
3\end{array}$ \\
\hline
\end{tabular}

a $I$ is the height of the diffraction peaks.

$\mathrm{b}$ These peaks were too strong to be completely recorded on the X-ray pattern.

Neither indium nor antimony oxides form compounds with titania. The X-ray patterns for the mixtures $\mathrm{In}_{2} \mathrm{O}_{3}: 2 \mathrm{TiO}_{2}$ and $\mathrm{Sb}_{2} \mathrm{O}_{3}: 2 \mathrm{TiO}_{2}$ show only the presence of $\mathrm{TiO}_{2}$. The specimens had contracted and lost weight, indicating that the other oxide had volatilized and had not combined with titania. It was found that, if $\mathrm{In}_{2} \mathrm{O}_{3}$ or $\mathrm{Sb}_{2} \mathrm{O}_{3}$ reacts with a second oxide to yield a true compound, little or no material is lost in volatilization under the heating conditions used in these experiments.

\subsection{Stannates}

Both of the rare-earth oxides, lanthana and neodymia, form cubic compounds with stannic oxide. There are only a few small peaks in the X-ray diffraction powder patterns (table 1) that cannot be indexed on the basis of a fluorite-type structure with a unit-cell size of approximately $5 \mathrm{~A}$. However, these additional lines indicate that the true structure is that of the pyrochlore type with a cell size of approximately $10 \mathrm{~A}$. The diminished intensity of these excess lines, as compared to those of the titanates, is due to the small difference in the scattering power of the two metal ions, as shown in table 4 for $\mathrm{Nd}_{2} \mathrm{Sn}_{2} \mathrm{O}_{7}$. No other rare-earth stannates have been studied, but it is probable that many of them form cubic pyrochlore compounds. Also, it is evident, from the work of Padrow and Schusterius [5] that solid solutions of the rare-earth titanates and stannates can occur.
TABLE 5. X-ray powder diffraction data for the compound $\mathrm{Bi}_{2} \mathrm{O}_{3} .2 \mathrm{SnO}_{2}$

\begin{tabular}{|c|c|c|c|c|c|}
\hline$d$ & $I \mathrm{a}$ & $d$ & $I^{\mathrm{a}}$ & $d$ & $I_{\mathrm{a}}^{\mathrm{a}}$ \\
\hline $\begin{array}{l}\text { 6. } 2 \\
\text { 3. } 77 \\
3.61 \\
3.22 \\
3.08\end{array}$ & $\begin{array}{l}25 \\
14 \\
46 \\
27 \\
\text { ь } 100++++\end{array}$ & $\begin{array}{l}1.496 \\
1.428 \\
1.425 \\
1.419 \\
1.394\end{array}$ & $\begin{array}{r}7 \\
5 \\
6 \\
10 \\
7\end{array}$ & $\begin{array}{r}1.028 \\
1.006 \\
0.9964 \\
.9924 \\
.9814\end{array}$ & $\begin{array}{r}15 \\
4 \\
4 \\
4 \\
3\end{array}$ \\
\hline $\begin{array}{l}2.78 \\
2.67 \\
2.47 \\
\text { 2. } 45 \\
2.39\end{array}$ & $\begin{array}{c}21 \\
\text { b } 100+++ \\
15 \\
22 \\
8\end{array}$ & $\begin{array}{l}\text { 1. } 391 \\
\text { 1. } 372 \\
\text { 1. } 349 \\
\text { 1. } 336 \\
\text { 1. } 328\end{array}$ & $\begin{array}{r}9 \\
4 \\
8 \\
17 \\
6\end{array}$ & $\begin{array}{l}.9756 \\
.9641 \\
.9446 \\
.9338 \\
.9315\end{array}$ & $\begin{array}{l}3 \\
4 \\
5 \\
6 \\
5\end{array}$ \\
\hline $\begin{array}{l}\text { 2. } 35 \\
2.240 \\
\text { 2. } 181 \\
\text { 2. } 090 \\
\text { 2. } 065\end{array}$ & $\begin{array}{r}13 \\
10 \\
5 \\
5 \\
7\end{array}$ & $\begin{array}{l}\text { 1. } 296 \\
\text { 1. } 259 \\
\text { 1. } 253 \\
\text { 1. } 235 \\
1.226\end{array}$ & $\begin{array}{r}6 \\
4 \\
8 \\
7 \\
30\end{array}$ & $\begin{array}{l}.9277 \\
.9200 \\
.9168 \\
.9067 \\
.9033\end{array}$ & $\begin{array}{r}5 \\
4 \\
4 \\
6 \\
14\end{array}$ \\
\hline $\begin{array}{l}2.057 \\
1.993 \\
1.888 \\
1.868 \\
1.812\end{array}$ & $\begin{array}{c}11 \\
6 \\
\text { ь } 100++ \\
8 \\
21\end{array}$ & $\begin{array}{l}1.205 \\
1.195 \\
1.175 \\
1.173 \\
1.166\end{array}$ & $\begin{array}{r}9 \\
25 \\
7 \\
7 \\
6\end{array}$ & $\begin{array}{l}.8905 \\
.8817 \\
.8765 \\
.8705 \\
.8645\end{array}$ & $\begin{array}{l}9 \\
4 \\
4 \\
4 \\
5\end{array}$ \\
\hline $\begin{array}{l}1.807 \\
1.780 \\
1.763 \\
1.717 \\
1.690\end{array}$ & $\begin{array}{r}16 \\
11 \\
14 \\
16 \\
8\end{array}$ & $\begin{array}{l}1.161 \\
1.148 \\
1.139 \\
1.122 \\
1.110\end{array}$ & $\begin{array}{l}5 \\
6 \\
4 \\
5 \\
6\end{array}$ & $\begin{array}{l}.8584 \\
.8480 \\
.8449 \\
.8374 \\
.8346\end{array}$ & $\begin{array}{l}5 \\
6 \\
8 \\
5 \\
5\end{array}$ \\
\hline $\begin{array}{l}1.629 \\
1.610 \\
1.597 \\
1.542 \\
1.531\end{array}$ & $\begin{array}{c}9 \\
\text { ь } 100+ \\
12 \\
32 \\
13\end{array}$ & $\begin{array}{l}1.091 \\
1.075 \\
1.054 \\
1.045 \\
1.033\end{array}$ & $\begin{array}{r}15 \\
5 \\
4 \\
4 \\
9\end{array}$ & $\begin{array}{l}.8242 \\
.8171 \\
.8149 \\
.8054 \\
.7987\end{array}$ & $\begin{array}{l}3 \\
8 \\
7 \\
6 \\
8\end{array}$ \\
\hline
\end{tabular}

a $I$ is the observed height of the diffraction peaks.'

$\mathrm{b}$ These peaks were too strong to be completely recorded on the X-ray pattern.

The reaction between $\mathrm{Bi}_{2} \mathrm{O}_{3}$ and $\mathrm{SnO}_{2}$ has been studied extensively by Coffeen $[13,14]$, who indicated that the hydrous bismuth stannate, $\mathrm{Bi}_{2-}$ $\left(\mathrm{SnO}_{3}\right)_{3} \cdot 5 \mathrm{H}_{2} \mathrm{O}$, fired at $1,149^{\circ} \mathrm{C}$, formed the compound $\mathrm{Bi}_{2}\left(\mathrm{SnO}_{3}\right)_{3}$. The present study indicates that the $\mathrm{Bi}_{2} \mathrm{O}_{3}: 3 \mathrm{SnO}_{2}$ mixture contains a new compound plus free $\mathrm{SnO}_{2}$, and that the composition $\mathrm{Bi}_{2} \mathrm{O}_{3} \cdot 2 \mathrm{SnO}_{2}$ contains no free $\mathrm{SnO}_{2}$. It had been reported by Aurivillius [15] that the system $\mathrm{Bi}_{2} \mathrm{O}_{3}-\mathrm{TiO}_{2}$ contained a compound, $\mathrm{Bi}_{4} \mathrm{Ti}_{3} \mathrm{O}_{12}$, that was pseudotetragonal, actually orthorhombic; therefore, the analogous compound $2 \mathrm{Bi}_{2} \mathrm{O}_{3}: 3 \mathrm{SnO}_{2}$ was looked for in the $\mathrm{Bi}_{2} \mathrm{O}_{3}-\mathrm{SnO}_{2}$ system. The reported structure could not be correlated with the compound found in the $\mathrm{Bi}_{2} \mathrm{O}_{3}-\mathrm{SnO}_{2}$ system. It must therefore be concluded that the $\mathrm{Bi}_{2} \mathrm{O}_{3}-\mathrm{SnO}_{2}$ compound is not isostructural with $\mathrm{Bi}_{2} \mathrm{Ti}_{3} \mathrm{O}_{12}$, but has a distorted pyrochlore-type structure with a ratio of $\mathrm{Bi}_{2} \mathrm{O}_{3}: 2 \mathrm{SnO}_{2}$.

The X-ray pattern of the $\mathrm{Bi}_{2} \mathrm{O}_{3} \cdot 2 \mathrm{SnO}_{2}$ compound given in table 5 resembles very strongly those of the cubic pyrochlores. All of the cubic peaks for a $10.68 \mathrm{~A}$ cubic pyrochlore structure can be found in this pattern, although there are other peaks present. A cubic unit cell of $21.37 \mathrm{~A}$ could account for most of these excess peaks, but some would be still unexplained. Although the pattern is very strongly pseudo-cubic, it must be concluded that the true symmetry is other than cubic.

It may be seen in figure 1 that the radius of the $\mathrm{Bi}^{+3}$ ion is close to that of $\mathrm{Gd}^{+3}$ and, on the basis of radius ratio only, could be expected to form a cubic pyrochlore structure with $\mathrm{SnO}_{2}$. However, the electronic polarization of the $\mathrm{Bi}^{+3}$ ion is probably different from that of the rare earths and may be 
related to the distortion from cubic symmetry. This notion of electronic polarization has been very valuable in explaining the distortion from cubic symmetry in the perovskite structures [16].

\subsection{Zirconates}

Specimens of $\mathrm{La}_{2} \mathrm{O}_{3}: 2 \mathrm{ZrO}_{2}$ and $\mathrm{Nd}_{2} \mathrm{O}_{3}: 2 \mathrm{ZrO}$ were carefully examined by X-ray diffraction powder analysis because previous work by Brown and Duwez [17] indicated that no compounds existed in these two systems. Table 1 shows that several extra lines of relatively low intensity were found in both patterns, which could be indexed only on the basis of a cubic pyrochlore-type compound. In table 4 the intensities calculated for $\mathrm{Nd}_{2} \mathrm{Zr}_{2} \mathrm{O}_{7}$, using an oxygen parameter of 0.414 , agree very well with the observed peak heights. A compound at a composition of $\mathrm{La}_{2} \mathrm{O}_{3}: 2 \mathrm{ZrO}_{2}$ was suggested by Trombe and Foex [18]. From the data presented it must be concluded that the compounds $\mathrm{La}_{2} \mathrm{Zr}_{2} \mathrm{O}_{7}$ and $\mathrm{Nd}_{2} \mathrm{Zr}_{2} \mathrm{O}_{7}$ do indeed exist and probably have a wide solid-solution range. They would fall in the middle of the stabilized cubic zirconia solid-solution regions, shown in the phase diagrams of the systems $\mathrm{La}_{2} \mathrm{O}_{3}-\mathrm{ZrO}_{2}$ and $\mathrm{Nd}_{2} \mathrm{O}_{3}-\mathrm{ZrO}_{2}$, proposed by Brown and Duwez [17] and reproduced here as figures 2 (a) and 3 (a). These compounds are indicated in the proposed revision of the two phase diagrams, figures 2 (b) and (c), and 3 (b). The compounds $\mathrm{La}_{2} \mathrm{O}_{3} \cdot 2 \mathrm{ZrO} \mathrm{O}_{2}$ and $\mathrm{Nd}_{2} \mathrm{O}_{3} \cdot 2 \mathrm{ZrO}_{2}$ have been indicated as melting congruently in figures 2 (b) and 3 (b) for the purpose of simplicity of the phase diagram, although no information is available on the melting points. If the compound melted incongruently, there could be no field of $\mathrm{La}_{2} \mathrm{Zr}_{2} \mathrm{O}_{7}$ (or $\mathrm{Nd}_{2} \mathrm{Zr}_{2} \mathrm{O}_{7}$ ) solid solution on the high $\mathrm{ZrO}_{2}$ side of the compound. This would not agree with the observed widening of the single-phase cubic solidsolution area shown by Brown and Duwez [17]. Figure 2 (c) indicates the phase equilibria in the $\mathrm{La}_{2} \mathrm{O}_{3}-\mathrm{ZrO}_{2}$ system if the pyrochlore compound actually melted incongruently.

It might be thought possible to have both a compound and a cubic $\mathrm{ZrO}_{2}$ solid solution in the phase diagrams. Such a situation would demand that the diffraction peaks in the X-ray patterns of the fluorite-type solid-solution phases coincided with the strong peaks of the pyrochlore compound, so as to agree with the observed single-phase solid solution. A highly complex diagram of this sort could be drawn for either a congruently or an incongruently melting compound. As a complexity of such a nature is unlikely, it is thought that the phase diagrams, figures 2 (b) and 3 (b), best fit all of the observed data.

It has been claimed by Ruff and Ebert [19], and Duwez, Brown, and Odel [20] that $\mathrm{Sc}_{2} \mathrm{O}_{3}, \mathrm{Sm}_{2} \mathrm{O}_{3}$, $\mathrm{Gd}_{2} \mathrm{O}_{3}$, and $\mathrm{Y}_{2} \mathrm{O}_{3}$ all form cubic zirconia solid solutions. These conclusions have been quoted by Dietzel and Tober [21]. In the present study, only the $\mathrm{Y}_{2} \mathrm{O}_{3}: 2 \mathrm{ZrO}_{2}$ composition has been examined. This specimen is apparently that of a singlephase fluorite material with no additional lines observable.

It is possible that the $\mathrm{Y}_{2} \mathrm{O}_{3}: 2 \mathrm{ZrO}_{2}$ composition is a true pyrochlore compound in which the faint
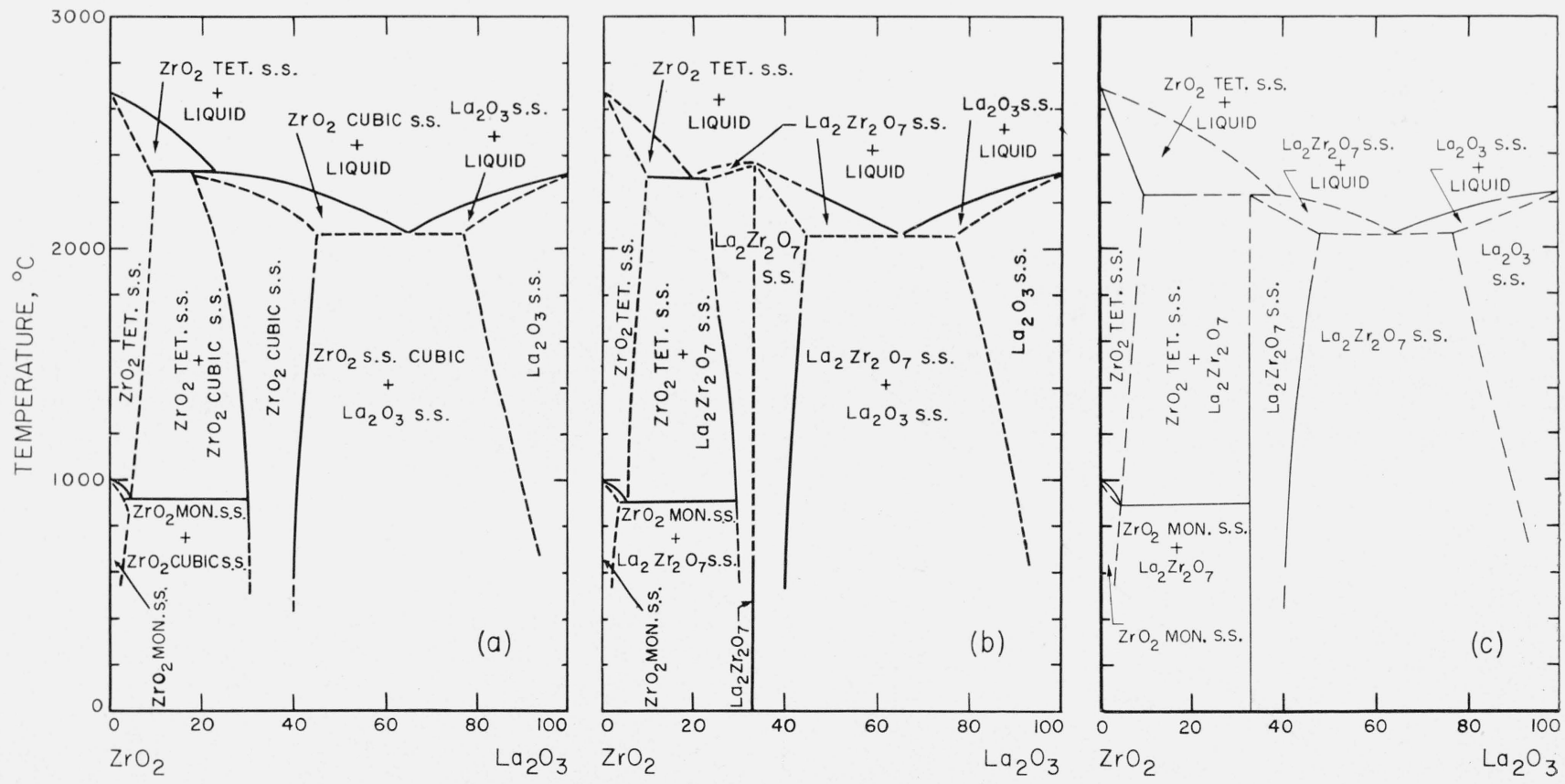

MOLE PERCENT

FIgure 2. Possible phase equilibria in lanthana-zirconia system.

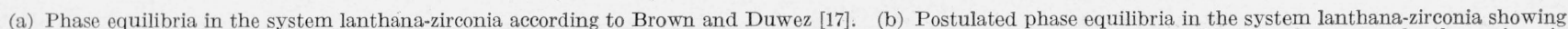

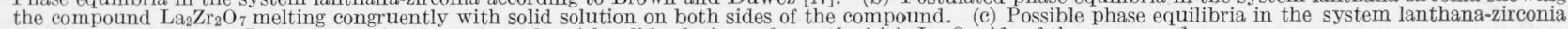
showing the compound $\mathrm{La}_{2} \mathrm{Zr}_{2} \mathrm{O}_{7}$ melting incongruently with solid solution only on the high $\mathrm{La}_{2} \mathrm{O}_{3}$ side of the compound. 


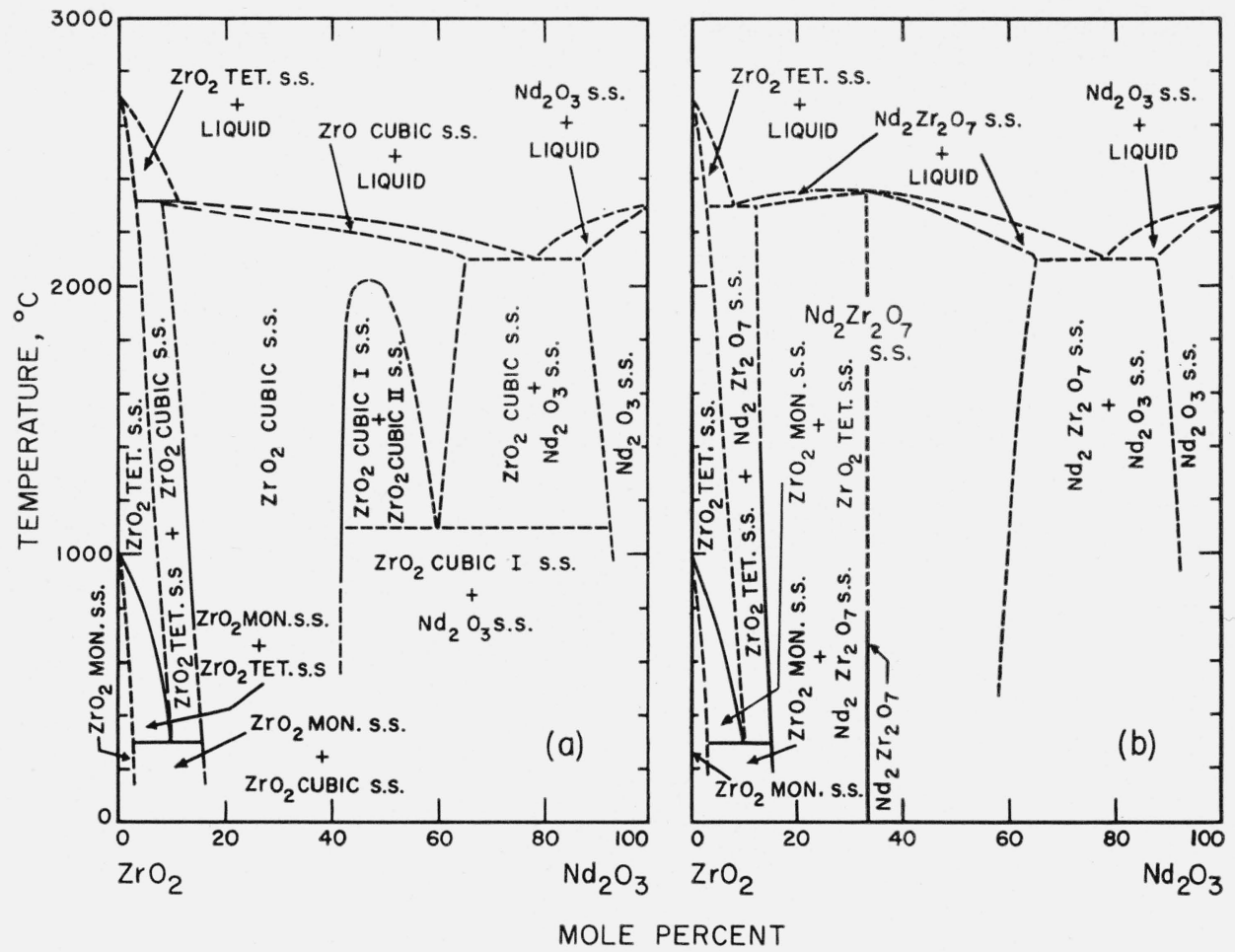

Figure 3. Possible phase equilibria in the neodymia-zirconia system.

(a) Phase equilibria in the system neodymia-zirconia according to Brown and Duwez [17]. (b) Postulated phase equilibria in the system neodymia-zirconia showing the compound $\mathrm{Nd}_{2} \mathrm{Zr}_{2} \mathrm{O}_{7}$ melting congruently with solid solution on both sides of the compound.

lines characteristic of pyrochlore are too weak to be observed. As shown in table 4, the intensity of these lines is almost entirely due to the difference in the scattering power of the $\mathrm{A}$ and $\mathrm{B}$ ions. The relative intensity of the additional peaks should be very small as the scattering factors of $\mathrm{Y}^{+3}$ and $\mathrm{Zr}^{+4}$ are almost exactly equal and the intensities depend almost entirely on the oxygen ions. The observed peak heights and the calculated intensities of $\mathrm{Y}_{2} \mathrm{Zr}_{2} \mathrm{O}_{7}$ show good correlation. Although it cannot be unequivocally decided whether the $\mathrm{Y}_{2} \mathrm{O}_{3}: 2 \mathrm{ZrO}_{2}$ composition is a fluorite solid solution or a pyrochlore-type compound, the latter is more probable, and it is not surprising that the excess lines cannot be seen on the X-ray diffraction powder patterns. It is, therefore, proposed that no stabilized cubic $\mathrm{ZrO}_{2}$ solid solutions exist in mixtures of $\mathrm{ZrO}_{2}$ and the larger trivalent ions. All such reported occurrences would than be solid solutions of $\mathrm{A}_{2} \mathrm{~B}_{2} \mathrm{O}_{7}$ pyrochlore compounds, as shown in figures 2 (b) and 3 (b).

The $\mathrm{In}_{2} \mathrm{O}_{3}: 2 \mathrm{ZrO}_{2}$ composition also shows only a single cubic fluorite-type phase in the X-ray diffraction powder pattern. As the $\mathrm{In}_{2} \mathrm{O}_{3}-\mathrm{ZrO}_{2}$ system is known to contain two phases in this area [22], and the present specimen contracted considerably and lost weight, it is assumed that the excess $\operatorname{In}_{2} \mathrm{O}_{3}$ was volatilized and that a cubic $\mathrm{ZrO}_{2}$ solid solution was actually found in this case.
A general rule can now be given for the reaction of $\mathrm{ZrO}_{2}$ with various oxides of trivalent metals:

Solid-state reactions of $\mathrm{ZrO}_{2}$ with oxides of the smaller trivalent ions, for example $\operatorname{In}_{2} \mathrm{O}_{3}$, yield cubic $\mathrm{ZrO}_{2}$ solid solutions; however, the larger trivalent ions probably result in $\mathrm{A}_{2} \mathrm{~B}_{2} \mathrm{O}_{7}$ pyrochlore-type solid solutions.

\subsection{Cerates and Uranates}

\section{a. Cerates}

The only cerate composition examined in this study was $\mathrm{In}_{2} \mathrm{O}_{3}: 2 \mathrm{CeO}_{2}$. The $\mathrm{X}$-ray pattern showed only $\mathrm{CeO}_{2}$ with no solid-solution or compound formation. The $\operatorname{In}_{2} \mathrm{O}_{3}$ was believed to have volatilized.

\section{b. Uranates}

Only two compositions containing $\mathrm{UO}_{2}$, applicable to the present work, $\mathrm{Nd}_{2} \mathrm{O}_{3}: 2 \mathrm{UO}_{2}$ and $\mathrm{Y}_{2} \mathrm{O}_{3}: 2 \mathrm{UO}_{2}$, were examined. These preparations have been described elsewhere [23] and will be reviewed briefly here. The $\mathrm{Y}_{2} \mathrm{O}_{3}: 2 \mathrm{UO}_{2}$ composition was definitely two phases, showing partial solid solution of both components. The X-ray pattern of the $\mathrm{Nd}_{2} \mathrm{O}_{3}: 2 \mathrm{UO}_{2}$ composition showed a single phase, apparently a fluorite-type solid solution (table 1). In this case the characteristic lines of the pyrochlore structure should easily have been observed, as $\mathrm{Nd}$ 
has an atomic number of 60 , whereas $\mathrm{U}$ is 92 . When the oxygen positions were shifted considerably from those of the other pyrochlores in an attempt to account for the zero intensity of the additional peaks, improbable uranium to oxygen bond lengths resulted. It must be concluded, therefore, that a compound of the type $\mathrm{Nd}_{2} \mathrm{U}_{2} \mathrm{O}_{7}$ probably does not exist and that the composition $\mathrm{Nd}_{2} \mathrm{O}_{3}: 2 \mathrm{UO}_{2}$ is a single-phase fluorite-type solid solution with an oxygen deficiency.

\section{Summary}

X-ray diffraction powder data have been presented to show that many $\mathrm{A}_{2} \mathrm{O}_{3}: 2 \mathrm{BO}_{2}$ compositions yield pyrochlore-type compounds when heated to the appropriate temperatures to give solid-state reactions. Oxides of the largest $\mathrm{A}^{+3}$ ions with $\mathrm{TiO}_{2}$ give distorted pyrochlore structures, whereas most of the oxides of the large $\mathrm{A}^{+3}$ ions form cubic pyrochlore compounds with the tetravalent ions. Oxides of the smaller $\mathrm{A}^{+3}$ ions do not form compounds. This information is shown in a plot of the radius of the $\mathrm{A}^{+3}$ ions versus that of the $\mathrm{B}^{+4}$ ions. Here it can be seen that certain areas of this diagram enclose certain structural types of compounds, although exceptions may be found.

Because of the discovery of small peaks characteristic of the pyrochlore structure in the X-ray diffraction powder patterns of $\mathrm{La}_{2} \mathrm{O}_{3} \cdot 2 \mathrm{ZrO}_{2}$ and $\mathrm{Nd}_{2} \mathrm{O}_{3} \cdot 2 \mathrm{ZrO}_{2}$, these compositions have been called pyrochlore-type compounds. The presence of these compounds necessitates a revision of the published phase diagrams for these systems, and such revisions have been shown.

\section{References}

[1] W. R. Cook and H. Jaffe, Phys. Rev. 88, 1426 (1952).

[2] W. R. Cook and H. Jaffe, Phys, Rev. 89, 1297 (1953).

[3] A. Bystrom, Arkiv Kemi, Mineral. Geol. [A] 18 (1945).

[4] L. W. Coughanour, R. S. Roth, S. Marzullo, and F. E. Sennett, J. Research NBS 54, 191 (1955) RP2580.

[5] N. N. Padrow and C. Schusterius, Ber. deut. keram. Ges. 31, 391 (1954).

[6] W. H. Davenport, S. S. Kistler, W. N. Wheildon, and O. T. Whittemore, J. Am. Ceram. Soc. 33, 333 (1950).

[7] H. R. Gaertner, Jahrb. Min. Beil. Bd. [1] 61, (1930).

[8] O. Rosen and A. Westgren, Geol. Fören. Förh. 60, 226 (1938)

[9] International tables for X-ray crystallography, vol. 1. (Kynoch Press, Birmingham, England, 1952).

[10] J. Green, Bul. Geol. Soc. Am. 64, 1001 (1953).

[11] E. A. Wood, Acta Cryst. 4, 353 (1951).

[12] M. L. Keith and R. Roy, Am. Mineralogist 39, 1 (1954).

[13] W. Coffeen, J. Am. Ceram. Soc. 36, 207 (1953).

[14] W. Coffeen (personal communication).

[15] B. Aurivillius; Ark. Kem. Sverige. 1, 499 (1950).

[16] S. Roberts, Phys. Rev. $\boldsymbol{7 6}, 1217$ (1949).

[17] F. H. Brown, Jr. and P. Duwez, J. Am. Ceram. Soc. 38, 95 (1955).

[18] F. Trombe and M. Foex, Compt. rend. 233, 254 (1951).

[19] O. Ruff, and F. Ebert, Z. anorg. u allgem. Chem. 180, 19 (1929).

[20] P. Duwez, F. H. Brown, Jr., and F. Odell, J. Electrochem. Soc. 38, 356 (1951).

[21] A. Dietzel and H. Tober, Ber. deut. keram. Ges. 30, 71 (1953).

[22] C. Schusterius and N. N. Padrow, Ber. deut. keram. Ges. 30, 235 (1953).

[23] S. M. Lang, F. P. Knudsen, R. S. Roth, and C. L. Filmore, NBS Circular 568 (1956).

Washington, September 21, 1955. 